\section{Rol de la cirugía bariátrica/metabólica en el manejo de la diabetes mellitus 2. Consenso SOCHED/SCCBM}

\author{
JORGE SAPUNAR ${ }^{1}$, ALEX ESCALONA ${ }^{2}$, A. VERÓNICA ARAYA ${ }^{3,4}$, \\ CARMEN GLORIA AYLWIN ${ }^{2,5}$, MARÍA JULIANA BASTÍAS ${ }^{6}$, \\ CAMILO BOZA ${ }^{4}$, CARLOS CÁRCAMO ${ }^{7}$, ATTILA CSENDES A. ${ }^{3}$, \\ PATRICIO DAVIDOFF ${ }^{8}$, RICARDO FUNKE ${ }^{4}$, PATRICIA GÓMEZ $Z^{3,4}$, \\ MARÍA ISABEL GONZÁLEZ ${ }^{9}$, RODOLFO LAHSEN ${ }^{4}$, \\ ENRIQUE LANZARINI ${ }^{3}$, ALBERTO MAÍZ ${ }^{10}$, \\ VERÓNICA MUJICA ${ }^{11}$, RODRIGO MUÑOZ ${ }^{12}$, GUSTAVO PÉREZ ${ }^{12}$, \\ FÉLIX RAIMANN $^{13}$, PATRICIO SALMAN ${ }^{14}$, \\ MATÍAS SEPÚLVEDA ${ }^{5}$, NÉSTOR SOTO ${ }^{15}$, RODRIGO VILLAGRÁN ${ }^{16}$
}

\section{Consensus statement of the Chilean endocrinological society on the role of bariatric surgery in type 2 diabetes}

Diabetes Mellitus (DM) and obesity are a public health problem in Chile. Bariatric surgery is the most effective treatment alternative to achieve a significant and sustained weight reduction in patients with morbid obesity. The results of controlled clinical trials indicate that, compared to medical treatment, surgery for obese patients with DM2 allows a better control of blood glucose and cardiovascular risk factors, reduces the need for medications and increases the likelihood for remission. Consensus conferences and clinical practice guidelines support bariatric surgery as an option to treat DM2 in Class III Obesity (Body Mass Index (BMI) > 40) regardless of the glycemic control and the complexity of pharmacological treatment and in Class II Obesity (BMI 35-39,9) with inadequate glycemic control despite optimal pharmacological treatment and lifestyle. However, surgical indication for patients with DM2 and BMI between 30-34.9, the most prevalent sub-group, is only suggested. The Chilean Societies of Endocrinology and Diabetes and of Bariatric and Metabolic Surgery decided to generate a consensus regarding the importance of other factors related to DM2 that would allow a better selection of candidates for surgery, particularly when weight does not constitute an indication. Considering the national reality, we also need a statement regarding the selection and characteristics of the surgical procedure as well as the role of the diabetologist in the multidisciplinary team.

(Rev Med Chile 2018; 146: 1175-1183)

Key words: Bariatric Surgery; Consensus; Diabetes Mellitus, Type 2.
'Unidad de Endocrinología, Departamento de Medicina Interna y Centro EPICYN, Facultad de Medicina, Universidad de la

Frontera. Temuco, Chile.

${ }^{2}$ Facultad de Medicina, Universidad de los Andes. Clínica Universidad de los Andes. Santiago, Chile.

${ }^{3}$ Hospital Clínico de la Universidad de Chile. Santiago, Chile.

${ }^{4}$ Clínica Las Condes. Santiago, Chile.

${ }^{5}$ Hospital de la Dirección de Previsión de Carabineros de Chile (DIPRECA). Santiago, Chile. ${ }^{6}$ Sección Endocrinología, Diabetes y Nutrición. Departamento de Medicina Interna. Hospital Naval Almirante Nef. Viña del Mar,

Chile.

${ }^{7}$ Instituto de Cirugía, Facultad de Medicina, Universidad Austral de Chile. Valdivia, Chile.

${ }^{8}$ Hospital FACH. Santiago, Chile. ${ }^{9}$ Clínica Sanatorio Alemán. Concepción, Chile.

${ }^{10}$ Departamento. Nutrición,

Diabetes y Metabolismo. Escuela de Medicina Pontificia Universidad Católica de Chile. Santiago, Chile.

${ }^{11}$ Facultad de Medicina. Universidad Católica del Maule. Talca, Chile.

${ }^{12}$ Departamento de Cirugía Digestiva, Facultad de Medicina, Pontificia Universidad Católica de Chile. Santiago, Chile.

${ }^{13}$ Centro Integral de Obesidad y Diabetes. Servicio de Cirugía y Endoscopía. Clínica Puerto Varas. Puerto Varas, Chile.

${ }^{14}$ Unidad de Endocrinología, Departamento de Medicina Interna, Facultad de Medicina. Universidad de Concepción.

Concepción, Chile.

${ }^{15}$ Unidad de Endocrinología y Diabetes, Servicio de Medicina Interna, Hospital San Borja Arriarán. Santiago, Chile. ${ }^{16}$ Departamento de Cirugía Bariátrica Metabólica, Clínica Bupa Antofagasta. Antofagasta, Chile.

Los autores declaran no tener conflictos de interés. Trabajo no recibió financiamiento.

Correspondencia a: Dr. Jorge Sapunar Manuel Montt 112, Temuco, Región de La Araucanía, Chile. jorge.sapunar@ufrontera.cl 


\section{Introducción}

\section{Epidemiología de la Diabetes mellitus y trastornos nutricionales por exceso en Chile}

\section{L} a diabetes mellitus (DM) es uno de los principales problemas de salud pública por su alta prevalencia y morbimortalidad asocia$\mathrm{da}^{1}$. En Chile, la proporción de personas mayores de 15 años con DM ha aumentado de 6,4\% el año 2003 a 12,3\% en la actualidad (Encuesta Nacional de Salud, ENS 2016-2017)2. Esta alarmante cifra en gran parte refleja el fracaso en las medidas de prevención y constituye un desafío para los sistemas de salud por la necesidad de ofrecer a los pacientes los recursos terapéuticos para mitigar las complicaciones crónicas de la enfermedad ${ }^{1}$. A pesar de las múltiples opciones disponibles para el tratamiento de la DM aún muchos pacientes no logran los objetivos de control para la enfermedad $^{3}$. La proporción de personas con DM que reportan HbAlc < 7\% en nuestro país aumentó de 34\% (ENS 2010) a 58\% (ENS 2016-2017), cifra muy similar a la de países desarrollados ${ }^{4}$. Aunque un adecuado control glicémico se asocia con reducción en la frecuencia de complicaciones microvasculares, la evidencia señala que para reducir la enfermedad cardiovascular, que es la principal causa de mortalidad en los pacientes con DM, deben controlarse simultáneamente la presión arterial y lípidos séricos 5 . En la ENS 2010 sólo 1,7\% de los pacientes con DM cumplían con estas 3 metas $^{6}$.

Entre las condiciones que dificultan el control de la diabetes mellitus 2 (DM2) está el sobrepeso y la obesidad, que afectan a una cada vez mayor proporción de personas de nuestro país ${ }^{2}$ y están presentes en la mayoría de los casos. Hay múltiples mecanismos involucrados en la interacción obesidad-diabetes que producen resistencia a la insulina en los tejidos periféricos y deficiencia en la secreción de la hormona por la célula beta pancreática, fenómenos que en conjunto llevan a hiperglicemia progresiva y desarrollo de $\mathrm{DM} 2^{7}$. La mantención de la obesidad durante el curso de la enfermedad perpetúa esta compleja interacción y está demostrado que el control del peso con terapias dietéticas, farmacológicas o quirúrgicas tienen un impacto favorable en el control glicémico e integral del paciente obeso con $\mathrm{DM}^{8}$.

\section{Cirugía bariátrica, obesidad y Diabetes mellitus 2}

La cirugía bariátrica (CB) es la alternativa de tratamiento más efectiva para lograr una reducción de peso significativa y sostenida a largo plazo en pacientes con obesidad mórbida ${ }^{9}$. Estudios de cohorte prospectivos con seguimiento de hasta 20 años demuestran que los pacientes sometidos a CB presentan un mejor control de las enfermedades asociadas a la obesidad y una reducción del riesgo de mortalidad a largo plazo comparado con pacientes no operados ${ }^{10}$. Efectivamente, la reducción del exceso de peso corporal obtenida con el tratamiento quirúrgico permite un mejor control de las enfermedades que constituyen factores de riesgo de morbilidad y mortalidad cardiovascular a largo plazo tales como DM2, hipertensión arterial y dislipidemias. En DM2, estos estudios demuestran una mejoría significativa y sostenida a largo plazo en el control de la enfermedad en 50 a $70 \%$ de los pacientes ${ }^{11}$. La mejoría en el control metabólico de la DM2 es directamente proporcional a la reducción de peso conseguida con diferentes procedimientos quirúrgicos.

Los resultados de ensayos clínicos controlados han evidenciado que el tratamiento quirúrgico de pacientes obesos con DM2 permite un mejor control de la glicemia y de los factores de riesgo cardiovascular, menor uso de medicamentos y mayores tasas de remisión comparado con las diferentes alternativas de tratamiento médico ${ }^{12-14}$.

Desde que se inicia la experiencia en el tratamiento quirúrgico de la obesidad, a mediados del siglo XX, son muchos los procedimientos y técnicas descritas y evaluadas, inicialmente con técnica abierta y posteriormente con abordaje laparoscópico. De los procedimientos descritos los más estudiados y validados son el bypass gástrico, gastrectomía vertical o en manga, banda gástrica ajustable y derivación biliopancreática (Figura 1), siendo la vía laparoscópica la alternativa de elección en la mayoría de los pacientes en la actualidad. De acuerdo a los datos de la serie clínica mundial de la International Federation for the Surgery of Obesity and Metabolic Disorders ${ }^{15}$ de 685.874 pacientes sometidos a cirugía primaria el año 2016, 53,6\% correspondieron a gastrectomía vertical y $34,9 \%$ a bypass gástrico. De acuerdo a la información del Comité de Registro de la SCCBM, la proporción de pacientes sometidos a gastrecto- 
A

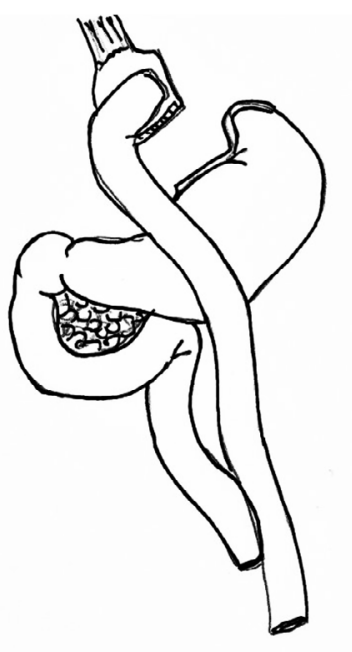

C

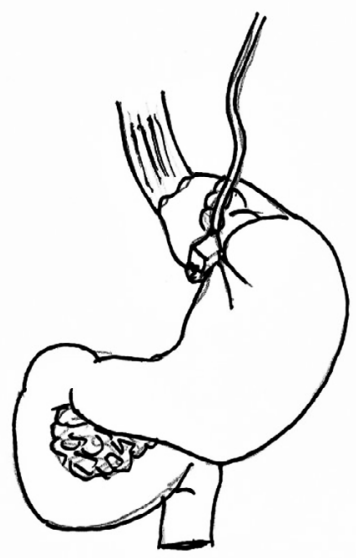

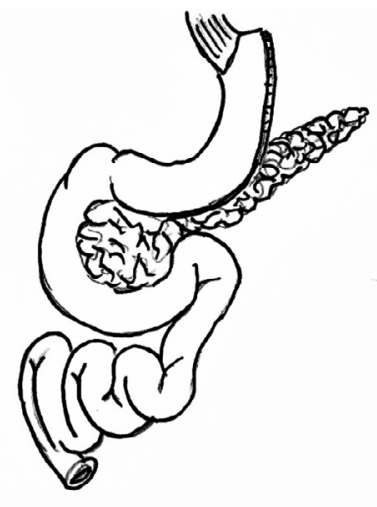

B

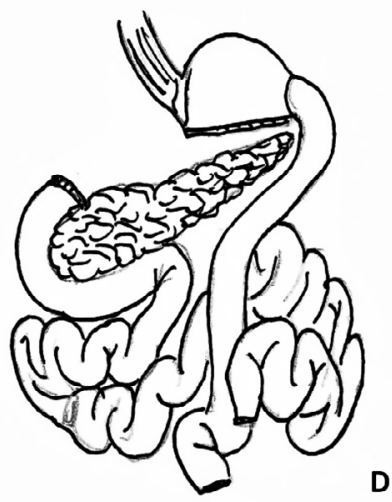

Figura 1. Procedimientos quirúrgicos utilizados en el tratamiento de la DM2. A: Bypass gástrico; B: Gastrectomía en manga; C: Banda inflable; D: Derivación bilio-pancreática. mía vertical y bypass gástrico durante el año 2017 es comparable a la reportada por la International Federation for the Surgery of Obesity and Metabolic Disorders (64\% y 26\% respectivamente), con una proporción de pacientes sometidos a banda gástrica ajustable, derivación biliopancreática o switch duodenal como procedimiento primario de $0,1 \%$ (Datos no publicados).

Históricamente, los procedimientos quirúrgicos para el tratamiento de la obesidad han sido clasificados en restrictivos, malabsortivos y mixtos, de acuerdo al mecanismo a través del cual ayudan a controlar el exceso de peso corporal.
Según esta clasificación la gastrectomía vertical sería considerada un procedimiento restrictivo y el bypass gástrico un procedimiento mixto ya que combina una restricción de la ingesta alimentaria con la confección de un estómago muy pequeño y la disminución de la absorción calórica por ausencia de contacto de los nutrientes en parte del intestino proximal. Estudios más recientes han demostrado que los cambios anatómicos asociados a los diferentes procedimientos quirúrgicos inducen cambios fisiológicos que serían en gran parte responsables del eficaz control del exceso de peso corporal. Estos mecanismos incluyen modifi- 
caciones en la secreción de hormonas gastrointestinales, en el metabolismo de ácidos biliares y en la microbiota intestinal entre otros. Estos cambios entero-hormonales serían especialmente importantes en el control de alteraciones metabólicas secundarias a la obesidad, destacando la mejoría en mecanismos reguladores de la homeostasis glucídica y, a largo plazo mantener el control del exceso de peso corporal ${ }^{16}$.

\section{Recomendaciones y sugerencias de conferencias de consenso y guías clínicas}

Considerando la importancia epidemiológica de la DM2, su fuerte vinculación con los trastornos nutricionales por exceso y la creciente evidencia que sustenta el rol de la cirugía en el tratamiento de esta enfermedad, no sorprende el desarrollo de múltiples conferencias para alcanzar un consenso en su indicación ${ }^{17-19}$. En la medida que se dispone de mayor evidencia de los beneficios de la cirugía de la obesidad en pacientes diabéticos con menor exceso de peso, el umbral ponderal de la recomendación quirúrgica en DM2 se ha ampliado a pacientes con IMC menores, surgiendo el concepto de cirugía metabólica ${ }^{16}$. A continuación revisaremos las recomendaciones y sugerencias de conferencias de consenso y guías clínicas en orden cronológico.

\section{a. Conferencias de consenso}

El año 2007 se realizó en Roma la "Primera Cumbre de Cirugía en Diabetes”, cuyo propósito fue elaborar una guía para la evaluación y el uso de cirugía en el tratamiento de la enfermedad ${ }^{17}$. Participaron 50 delegados y para lograr consenso se aplicó la metodología de panel de expertos. Las conclusiones se resumen en la Tabla 1, destacando que los criterios para selección de casos para cirugía entre los pacientes con DM2 fueron el IMC y la respuesta al tratamiento médico de la enfermedad.

El año 2011 The International Diabetes Federation Taskforce on Epidemiology and Prevention of Diabetes convocó un grupo de expertos, integrado por médicos diabetólogos, endocrinólogos, cirujanos bariátricos y expertos en salud pública, que mediante consenso desarrollaron recomendaciones para seleccionar pacientes obesos con DM2 para cirugía bariátrica/metabólica ${ }^{18}$. En conclusión el panel de expertos recomendó la cirugía como tratamiento para la DM2 en sujetos con IMC > 35, con mal control metabólico y manejo médico óptimo de la enfermedad. Bajo ciertas circunstancias serían elegibles para cirugía sujetos con IMC entre 30-35.

El año 2015 se realizó en Londres la "Segunda Cumbre de Cirugía en Diabetes" cuyo objetivo fue desarrollar una guía global para informar a los clínicos y responsables de políticas de salud sobre los beneficios y limitaciones de la cirugía en el tratamiento de la DM2 ${ }^{19}$. Luego de evaluar y resumir la evidencia disponible en MEDLINE, se realizaron 3 rondas de metodología Delphi para lograr consenso. Participaron 48 expertos, incluyendo representantes de las principales organizaciones en DM. Las conclusiones de la cumbre fueron respaldadas por 44 organizaciones y sociedades científicas, entre ellas las Sociedades Chilenas de Endocrinología y Diabetes (SOCHED) y de Cirugía Bariátrica y Metabólica (SCCBM). Las recomendaciones para la selección de pacientes se resumen en la Tabla 2 y para la elección del procedimiento quirúrgico en la Tabla 3 . Al igual que en la primera cumbre la selección de casos para cirugía entre los pacientes con DM2 fueron el IMC y la respuesta al tratamiento médico de la enfermedad.

\section{b. Guías clínicas para el manejo de la DM2}

Las principales guías clínicas para el manejo de la DM2 a nivel internacional, latinoamericano

Tabla 1. Recomendaciones y Sugerencias de la Primera Cumbre de Cirugía en Diabetes. Roma, 2007 17

- La cirugía debe ser considerada en el tratamiento de la DM2 en sujetos con IMC > 35 que cumplan con criterios de selección quirúrgicos (Grado A)

- La cirugía podría ser considerada como intervención de segunda línea en sujetos con IMC entre 30-35 con control inadecuado de la DM2 (Grado B)

- El bypass gástrico en Y de Roux podría ser una opción adecuada para el tratamiento de la DM2 (Grado C)

- Otras técnicas quirúrgicas deberían ser utilizadas sólo en ensayos clínicos aprobados por comités de ética (Grado A)

- Para mejorar la calidad de la evidencia es necesario desarrollar estándares para medir desenlaces clínicos y fisiológicos (Grado A)

Nivel de consenso: Grado C: $67-77 \%$. Grado B: $78-89 \%$. Grado A: 90-100\%. 
Rol de cirugía bariátrica/metabólica en manejo de la diabetes mellitus 2 - J. Sapunar et al

Tabla 2. Recomendaciones para la selección de pacientes con DM2 para cirugía. Segunda Cumbre de Cirugía en Diabetes. Londres, $2015^{19}$

- La selección de pacientes debe depender de un equipo multidisciplinario que incluiría al cirujano, médico internista o diabetólogo/endocrinólogo y nutricionista con experiencia en DM2 (Grado B)

- Contraindicaciones a la cirugía son DM1 (a menos que la indicación sea la obesidad), abuso de drogas o alcohol, enfermedad psiquiátrica no controlada, falta de comprensión de riesgo/beneficio, de los desenlaces esperados o alternativas, falta de compromiso con la suplementación nutricional y con el seguimiento a largo plazo requerido por la cirugía (Grado A)

- La cirugía metabólica es recomendada como una opción para tratar la DM2 (Grado U) en: Obesidad clase III (IMC $\geq 40$ ) independientemente del control glicémico y de la complejidad del tratamiento farmacológico. Obesidad clase II (IMC 35-39,9) con control glicémico inadecuado a pesar de tratamiento farmacológico y estilo de vida óptimos

- La cirugía metabólica podría ser considerada como una opción para tratar la DM2 en pacientes con obesidad tipo I (IMC 30-34,9) con control glicémico inadecuado a pesar de tratamiento farmacológico y estilo de vida óptimo (Grado B)

- La cirugía en adolescentes con DM2 no puede ser recomendada por falta de evidencia que sustente la indicación (Grado U)

Nivel de consenso: Grado C: 67-77\%. Grado B: 78-89\%. Grado A: 90-99\%. Grado U: 100\% acuerdo.

Tabla 3. Criterios para la elección del procedimiento quirúrgico en DM2. Segunda Cumbre de Cirugía en Diabetes. Londres, $2015^{19}$

- El bypass gástrico en Y de Roux tiene el perfil de riesgo/beneficio más favorable en la mayoría de los pacientes con DM2 (Grado U)

- Aunque se requieren estudios a largo plazo, la manga gástrica es un procedimiento efectivo para reducción de peso y mejoría de la DM2, al mediano plazo (Grado B)

- A pesar que la banda gástrica ajustable es efectiva en el tratamiento de la DM2 en pacientes con obesidad, tiene un riesgo aumentado de re-operación/revisión comparada con otras técnicas (Grado B)

- Aunque existe evidencia que la derivación bilio-pancreática es el procedimiento más efectivo en términos de control glicémico y pérdida de peso, su perfil de riesgo/beneficio es menos favorable y debe reservarse sólo para grados extremos de obesidad (Grado B).

Nivel de consenso: Grado C: 67-77\%. Grado B: 78-89\%. Grado A: 90-99\%. Grado U: 100\% acuerdo.

y nacional han considerado el rol de la cirugía bariátrica y metabólica ${ }^{8,20,21}$. En la Tabla 4 se resumen las recomendaciones y sugerencias de 3 guías de práctica clínica que expresan la fuerza de la recomendación por metodología GRADE y cuya calidad evaluada por AGREE ha ido en aumento. Es interesante comprobar que conceptualmente el rol de la cirugía ha cambiado desde el bariátrico 20,21 al metabólico ${ }^{8}$ a pesar de lo cual la indicación quirúrgica en pacientes con IMC entre 30-34,9 y con control glicémico deficiente sigue siendo sólo sugerida.

\section{Objetivo}

Después revisar las recomendaciones y sugerencias de conferencias de consenso y guías de práctica clínica disponibles en la literatura y respaldar la cirugía metabólica como una opción para tratar la DM2 en Obesidad clase III (IMC $>40$ ) independientemente del control glicémico y de la complejidad del tratamiento farmacológico y en Obesidad clase II (IMC 35-39,9) con control glicémico inadecuado a pesar de tratamiento farmacológico y estilo de vida óptimos, creemos que es necesario consensuar la importancia de otros factores relacionados con la DM2 que permitirían seleccionar en mejor forma pacientes para cirugía metabólica, particularmente cuando el IMC no constituye una indicación por sí misma. Considerando la realidad nacional también nos parece necesario un pronunciamiento respecto a la selección y características del procedimiento quirúrgico así como el rol del diabetólogo en el equipo multidisciplinario. 
Tabla 4. Recomendaciones de 3 Guías de Práctica Clínica para el Rol de la Cirugía Bariátrica/Metabólica en el Manejo de la DM2

\begin{tabular}{|c|c|c|}
\hline Guía clínica & Recomendación & $\begin{array}{l}\text { Fuerza } \\
\text { (GRADE) }\end{array}$ \\
\hline \multirow[t]{2}{*}{$\begin{array}{l}\text { Guía MINSAL } \\
2010^{20}\end{array}$} & $\begin{array}{l}\text { La cirugía es una alternativa en pacientes con DM2 e IMC > } 35 \text { que cumplan con los } \\
\text { criterios propios de dicha indicación }\end{array}$ & B \\
\hline & $\begin{array}{l}\text { La cirugía debe ser indicada y realizada por equipos médicos competentes que aseguren } \\
\text { riesgos controlados y capacitados para el manejo de complicaciones quirúrgicas y nutri- } \\
\text { cionales a corto y largo plazo }\end{array}$ & A \\
\hline \multirow[t]{5}{*}{$\begin{array}{l}\text { Guía ALAD } \\
2013^{21}\end{array}$} & $\begin{array}{l}\text { La cirugía es terapia de elección en pacientes con DM2 e IMC } \geq 40 \text { que cumplan con los } \\
\text { criterios propios de dicha indicación }\end{array}$ & A \\
\hline & $\begin{array}{l}\text { La cirugía puede ser recomendada en pacientes con } \mathrm{DM} 2 \text { e IMC } \geq 35 \text {, cuando no se logra } \\
\text { control de la glicemia con cambios en estilo vida y terapia farmacológica }\end{array}$ & B \\
\hline & $\begin{array}{l}\text { La cirugía puede ser considerada en pacientes con DM2 e IMC entre } 30 \text { y } 35 \text { si el control } \\
\text { glicémico es difícil a pesar de manejo óptimo e intensivo y si hay comorbilidades adicio- } \\
\text { nales a la DM2 }\end{array}$ & B \\
\hline & No se recomienda la cirugía bariátrica en pacientes con DM2 e IMC $<30$ & $\mathrm{E}$ \\
\hline & $\begin{array}{l}\text { Los pacientes candidatos a cirugía bariátrica deben ser tratados e intervenidos en centros } \\
\text { multidisciplinarios certificados }\end{array}$ & $\mathrm{E}$ \\
\hline \multirow[t]{3}{*}{$\begin{array}{l}\text { Guía ADA } \\
2018^{8}\end{array}$} & $\begin{array}{l}\text { La cirugía debe ser recomendada en pacientes con DM2 e IMC } \geq 40 \text { ( } 37,5 \text { en asiáticos) } \\
\text { independientemente del control glicémico y complejidad del tratamiento, con IMC 35- } \\
39,9 \text { ( } 32,5-37,4 \text { en asiáticos) si el control glicémico es difícil a pesar de manejo óptimo e } \\
\text { intensivo. Deben candidatos quirúrgicos adecuados }\end{array}$ & A \\
\hline & $\begin{array}{l}\text { La cirugía debe ser considerada en pacientes con DM2 e IMC 30- 34,9 (27,5-32,4 en } \\
\text { asiáticos) si el control glicémico es difícil a pesar de manejo óptimo e intensivo. Deben } \\
\text { candidatos quirúrgicos adecuados }\end{array}$ & B \\
\hline & $\begin{array}{l}\text { La cirugía debe ser realizada en centros con alto flujo de pacientes, con equipos multidis- } \\
\text { ciplinarios con experiencia en DM2 y cirugía digestiva }\end{array}$ & C \\
\hline
\end{tabular}

\section{Metodología}

La Sociedad Chilena de Endocrinología y Diabetes (SOCHED) y la Sociedad Chilena de Cirugía Bariátrica y Metabólica (SCCBM) designaron 2 delegados para coordinar un pronunciamiento conjunto. Este equipo de coordinación seleccionó a 20 expertos nacionales ( 10 médicos diabetólogos y 10 médicos cirujanos), certificados, con reconocimiento y experiencia en el área de interés. El grupo de expertos en diabetología fue sometido a un proceso Delphi para lograr consenso en criterios de selección de pacientes y los expertos en cirugía para elección de la técnica quirúrgica. Ambos grupos, también por metodología Delphi, buscaron consenso en el rol del diabetólogo en el equipo multidisciplinario a cargo del manejo quirúrgico de la DM2.

Para cada aspecto en discusión se elaboraron afirmaciones acompañadas de una escala tipo Likert $^{22}$ con 5 opciones, en la que los expertos expresaron su grado de acuerdo. Definimos consenso cuando más del $66 \%$ de los expertos estuvieron de acuerdo o en desacuerdo con la afirmación. El nivel de consenso se clasificó en grado C (67-77\%), grado B (78-89\%), grado A $(90-99 \%)$ y grado U (100\%). Con nivel de consenso U-A el panel de expertos recomendó ejecutar o no ejecutar la acción implícita en la afirmación. Con nivel de consenso B-C el panel sólo sugirió.

El contenido del pronunciamiento fue sometido a la revisión de los Directorios de SOCHED y SCCBM. 
Rol de cirugía bariátrica/metabólica en manejo de la diabetes mellitus 2 - J. Sapunar et al

\section{Recomendaciones y Sugerencias}

\section{Criterios de Selección de Pacientes con DM2 para Cirugía Bariátrica y/o Metabólica}

\section{Objetivo terapéutico remisión de la DM2}

En la Tabla 5 se detallan los criterios para seleccionar pacientes con DM2 para tratamiento quirúrgico cuando el objetivo terapéutico es la remisión. Los pacientes a tamizar tendrían un IMC entre 30 y 34,9 con mal control metabólico y tratamiento óptimo de la enfermedad.

- Se sugiere, en la mayor parte de los casos, hablar de mejor control de la enfermedad que remisión como objetivo terapéutico de la cirugía metabólica en pacientes con DM2 (Grado de Consenso C).

- Se recomienda la opción quirúrgica para lograr remisión de la enfermedad (Criterio ADA) cuando la DM2 tenga menos de 10 años de evolución (Grado de Consenso U).

- Se sugiere no descartar la cirugía metabólica en enfermedades con menos de un año de evolución, aunque no sea posible reconocer en tan corto plazo aquellas que evolucionarán a dependencia a insulina (Grado de Consenso C).

- Se recomienda, antes de considerar la cirugía metabólica, descartar diabetes secundarias y por autoinmunidad como DM1 o LADA cuya mejoría o remisión con cirugía no está demostrada (con Auto-anticuerpos positivos) (Grado de Consenso U).

- Se recomienda considerar cirugía metabólica con un valor de péptido $\mathrm{C} \geq 1 \mathrm{ng} / \mathrm{ml}$ obtenido con $\mathrm{HbA1c}<8,5 \%$ como mínimo, aunque si el objetivo es la remisión es recomendable un péptido C con un valor $>3,8 \mathrm{ng} / \mathrm{ml}$ (Grado de Consenso U).

- A menor edad existe mayor probabilidad de remisión, particularmente cuando el paciente es menor de 40 años. En general sobre 65 años este objetivo no se puede lograr (Grado de Consenso U).

- Tanto la insulinoterapia como el uso de múltiples agentes antidiabéticos para mantener el control metabólico de la DM2 reflejan menor capacidad beta-insular y menor probabilidad de remisión de diabetes (Grado de Consenso $\mathrm{U})$.
- Son buenos candidatos a cirugía metabólica aquellos pacientes con DM2 que previamente han demostrado buena adherencia a sus controles, cumplen indicaciones y que no buscan la cirugía solo como una forma de eliminar restricciones, medicamentos o controles médicos (Grado de Consenso A).

\section{Objetivo terapéutico evitar progresión de complicaciones crónicas}

En la Tabla 6 se detallan los criterios para seleccionar pacientes con DM2 para tratamiento quirúrgico cuando el objetivo terapéutico es evitar la progresión de complicaciones crónicas

Tabla 5. Criterios de selección de pacientes con DM2 para cirugía cuando el objetivo terapéutico es la remisión de la enfermedad

\begin{tabular}{|l|}
\hline Criterio \\
Tiempo de evolución de la enfermedad \\
Resistencia a la insulina vs insuficiencia $\beta$-insular \\
Nivel de péptido C \\
Edad \\
Características del tratamiento médico de la DM2 \\
Presencia de co-morbilidades \\
Actitud y perfil psicológico del paciente \\
\hline
\end{tabular}

Se aplica a pacientes con IMC entre 34,9 y 30 (32,9 y 27 para asiáticos) con mal control metabólico y tratamiento óptimo de la enfermedad.

\section{Tabla 6. Criterios de selección de pacientes con DM2 para cirugía cuando el objetivo terapéutico es la prevención de complicaciones crónicas}

\begin{tabular}{|l|}
\hline Criterio \\
Riesgo cardiovascular aumentado \\
Presencia de nefropatía \\
Presencia de retinopatía \\
Hipertensión arterial severa \\
Presencia de esteatosis hepática \\
Presencia de síndrome de apnea/hipo-apnea obstructiva \\
del sueño \\
\hline Edad \\
\hline
\end{tabular}

Se aplica a pacientes con IMC entre 34,9 y 30 (32,9 y 27 para asiáticos) con mal control metabólico y tratamiento óptimo de la enfermedad. 
de la DM2. Los pacientes a tamizar tendrían un IMC entre 30 y 34,9 con mal control metabólico y manejo óptimo de la enfermedad.

- La cirugía metabólica en DM2 está asociada con reducción del riesgo y muerte cardiovascular a largo plazo (Grado de Consenso B).

- Se sugiere no indicar cirugía metabólica en pacientes con DM2 en prevención secundaria de eventos cardiovasculares, incluyendo aquellos con re-vascularización coronaria previa (Grado de Consenso C).

- La cirugía metabólica puede reducir la micro-albuminuria en pacientes con DM2, sin embargo no se sugiere en etapas avanzadas de nefropatía diabética, ya que puede aumentar la proteinuria y ocurrir un deterioro de la función renal (Grado de Consenso B).

- No hay consenso en los valores de relación albuminuria/creatininuria y depuración renal de creatinina requeridos para indicar o contraindicar la cirugía metabólica en DM2.

- La evidencia que respalda el tratamiento quirúrgico de la DM2 para evitar la progresión de la retinopatía es de baja calidad. En algunos casos la cirugía ha contribuido a la progresión de esta complicación crónica, especialmente con grados avanzados de retinopatía (Grado de Consenso C).

- No hay consenso si la presencia de hipertensión arterial severa o moderada a pesar de tratamiento médico adecuado podría justificar por si sola la cirugía metabólica en pacientes con DM2 e IMC entre 30 y 34,9.

- La esteatosis hepática es extraordinariamente frecuente en pacientes obesos con DM2 y podría ser considerada una razón para elegir la cirugía, sin embargo, se recomienda cautela en formas más avanzadas como esteato-hepatitis y cirrosis hepática (Grado de Consenso U).

- El síndrome de apnea/hipo-apnea obstructiva del sueño está fuertemente relacionado con la obesidad y se suma a otros factores para aumentar el riesgo cardiovascular. Su presencia en pacientes con DM2 e IMC entre 30 y 34,9 favorece una conducta quirúrgica (Grado de Consenso C).

- Si una complicación crónica de la DM2, en un paciente con IMC entre 30 y 34,9, está respondiendo adecuadamente a las medidas habituales no sería recomendable la cirugía. Por el contrario, si la complicación está progresando a pesar del tratamiento o bien el tratamiento es mal tolerado o está contraindicado, podría considerarse la cirugía (Grado de Consenso C).

\section{Selección del procedimiento quirúrgico}

- Se sugiere seleccionar la técnica en cirugía metabólica de DM2 de acuerdo al IMC, control metabólico, nivel de péptido $\mathrm{C}$, duración de la enfermedad y modalidad de tratamiento médico (Grado de Consenso B).

- Se sugiere el bypass gástrico como técnica de elección para cirugía metabólica en pacientes con DM2 (Grado de Consenso C).

- Se sugiere en bypass gástrico uniformar la longitud de las asas biliar y alimentaria (Grado de Consenso B).

- No hay consenso si la gastrectomía vertical deba ser considerada la alternativa de elección al bypass gástrico en cirugía metabólica de pacientes con DM2.

- Se sugiere en gastrectomía vertical uniformar la técnica quirúrgica, particularmente el tamaño de sonda de calibración (Grado de Consenso B).

\section{Equipo Multidisciplinario}

a. Se sugiere que todo paciente con DM que el equipo multidisciplinario considere candidato a cirugía bariátrica o metabólica sea evaluado por diabetólogo para confirmar la indicación, realizar manejo peri-operatorio y seguimiento (Grado de Consenso B).

b. Toda institución que realice cirugía metabólica en pacientes con DM debe incluir en su equipo multidisciplinario un médico diabetólogo certificado (Grado de Consenso A).

c. Todo equipo quirúrgico que realice cirugía metabólica en pacientes con DM debe tener experiencia en bypass gástrico (Grado de Consenso U).

\section{Referencias}

1. International Diabetes Federation. IDF Diabetes Atlas. 8th ed. 2017. Disponible en http://www.diabetesatlas. org/ (Consultado el 15 de julio de 2018).

2. Encuesta Nacional de Salud 2016-2017. Departamento 
de Epidemiología División de Planificación Sanitaria Subsecretaría de Salud Pública 2017; Disponible en: http://www.minsal.cl/wp-content/uploads/2017/11/ ENS-2016-17_PRIMEROS RESULTADOS.pdf

3. Lipska K, Yao X, Herrin J, McCoy R, Ross J, Steinman $\mathrm{M}$, Inzucchi S, et al. Trends in Drug Utilization, Glycemic Control, and Rates of Severe Hypoglycemia, 20062013. Diabetes Care 2017; 40: 468-75.

4. Informe Encuesta Nacional de Salud 2016-2017 Diabetes Mellitus. Departamento de Epidemiología, División de Planificación Sanitaria Ministerio de Salud. Disponible en: http://www.minsal.cl/wp-content/uploads/2017/11/ ENS-2016-17_PRIMEROS-RESULTADOS.pdf

5. American Diabetes Association. Cardiovascular disease and risk management: Standards of Medical Care in Diabetes 2018. Diabetes Care 2018; 41 (Suppl. 1): S86-S104.

6. Estrategia Nacional de Salud para el cumplimiento de los Objetivos Sanitarios de la Década 2011-2020. OE2 Crónicas no transmisibles: 50-60. Disponible en http://www.bibliotecaminsal.cl/wp/wp-content/ uploads/2011/12/Metas-2011-2020.pdf

7. Park S, Sadanala KC, Kim EK. A Metabolomic Approach to Understanding the Metabolic Link between Obesity and Diabetes Mol. Cells 2015; 38 (7): 587-96.

8. American Diabetes Association. Obesity Management for the Treatment of Type 2 Diabetes: Standards of Medical Care in Diabetes 2018. Diabetes Care 2018; 41: S65-S72.

9. Busetto L, Dicker D, Azran C, Batterham RL, Farpour-Lambert N, Fried M, et al. Practical Recommendations of the Obesity Management Task Force of the European Association for the Study of Obesity for the Post-Bariatric Surgery Medical Management. Obes Facts 2017; 10: 597-632.

10. Adams TD, Davidson LE, Litwin SE, Kolotkin RL, La Monte MJ, Pendleton RC, et al. Health benefits of gastric bypass surgery after 6 years. JAMA 2012; 308: 1122-31.

11. Courcoulas AP, King WC, Belle SH, Berk P, Flum DR, Garcia L, et al. Seven-Year Weight Trajectories and Health Outcomes in the Longitudinal Assessment of Bariatric Surgery (LABS) Study. JAMA Surg 2018; 153: 427-34.

12. Schauer PR, Bhatt DL, Kirwan JP, Wolski K, Aminian
A, Brethauer SA et al. Bariatric Surgery versus Intensive Medical Therapy for Diabetes - 5-Year Outcomes. N Engl J Med 2017; 376: 641-51.

13. Mingrone G, Panunzi S, De Gaetano A, Guidone C, Laconelli A, Nanni G, et al. Bariatric-metabolic surgery versus conventional medical treatment in obese patients with type 2 diabetes: 5 year follow-up of an open-label, single-centre, randomised controlled trial. Lancet 2015; 386: 964-73.

14. Courcoulas AP, Belle SH, Neiberg RH, Pierson SK, Eagleton JK, Kalarchian MA, et al. Three-Year Outcomes of Bariatric Surgery vs Lifestyle Intervention for Type 2 Diabetes Mellitus Treatment: A Randomized Clinical Trial. JAMA Surg 2015; 150: 931-40.

15. Angrisani L, Santonicola A, Iovino P, Vitiello A, Higa K, Himpens J, Buchwald H, Scopinaro N IFSO Worldwide Survey 2016: Primary, Endoluminal, and Revisional Procedures. Obes Surg 2018; 14: 553-12.

16. Batterham RL, Cummings DE. Mechanisms of diabetes improvement following bariatric/metabolic surgery. Diabetes Care 2016; 39: 893-901.

17. Rubino F, Kaplan LM, Schauer PR, Cummings DE. The Diabetes Surgery Summit Consensus Conference. Recomendations for the evaluation and use of gastrointestinal surgery to treat type 2 diabetes mellitus. Ann Surg 2010; 251: 399-405.

18. Dixon JB, Zimmet P, Alberti KG, Rubino F. Bariatric surgery: an IDF statement for obese Type 2 Diabetes. Diabet Med 2011; 28: 628-42.

19. Rubino F, Nathan DM, Eckel RH, Schauer PR, Alberti $\mathrm{KG}$, Zimmet PZ, et al. Metabolic surgery in the treatment algorithm for type 2 diabetes: A Joint Statement by International Diabetes Organization. Diabetes Care 2016; 39: 861-77.

20. Guía Clínica 2010 Diabetes Mellitus tipo 2. Disponible en: http://www.minsal.cl/portal/url/item/72213ed52c3e23d1e04001011f011398.pdf.

21. Guías ALAD sobre el Diagnóstico, Control y Tratamiento de la Diabetes Mellitus Tipo 2 con Medicina Basada en Evidencia. Edición 2013. Disponible en: https://issuu. com/alad-diabetes/docs/guias_alad_2013.

22. Carifio J, Perla RJ. Ten common misunderstandings, misconceptions, persistent myths and urban legends about Likert Scales and Likert Response Formats and their antidotes. J Soc Sci 2007; 3: 106-16. 